\title{
Interferon-Gamma Release Assays versus Tuberculin Skin Testing for the Diagnosis of Latent Tuberculosis Infection: An Overview of the Evidence
}

\author{
A. Trajman, ${ }^{1,2}$ R. E. Steffen, ${ }^{3}$ and D. Menzies ${ }^{2}$ \\ ${ }^{1}$ Gama Filho University, 20740-900 Rio de Janeiro, RJ, Brazil \\ ${ }^{2}$ Montreal Chest Institute, McGill University, Montreal, QC, Canada H2X 2P4 \\ ${ }^{3}$ Federal University of Rio de Janeiro, 21941-913 Rio de Janeiro, RJ, Brazil \\ Correspondence should be addressed to A. Trajman; atrajman@gmail.com
}

Received 5 October 2012; Accepted 10 January 2013

Academic Editor: Jonathan Golub

Copyright (C) 2013 A. Trajman et al. This is an open access article distributed under the Creative Commons Attribution License, which permits unrestricted use, distribution, and reproduction in any medium, provided the original work is properly cited.

A profusion of articles have been published on the accuracy and uses of interferon-gamma releasing assays. Here we review the clinical applications, advantages, and limitations of the tuberculin skin test and interferon-gamma release assays and provide an overview of the most recent systematic reviews conducted for different indications for the use of these tests. We conclude that both tests are accurate to detect latent tuberculosis, although interferon-gamma release assays have higher specificity than tuberculin skin testing in BCG-vaccinated populations, particularly if BCG is received after infancy. However, both tests perform poorly to predict risk for progression to active tuberculosis. Interferon-gamma release assays have significant limitations in serial testing because of spontaneous variability and lack of a validated definition of conversion and reversion, making it difficult for clinicians to interpret changes in category (conversions and reversions). So far, the most important clinical evidence, that is, that isoniazid preventive therapy reduces the risk for progression to disease, has been produced only in tuberculin skin test-positive individuals.

\section{Introduction}

Tuberculosis (TB) is an important cause of morbidity and mortality worldwide [1]. Governmental and non-governmental organization efforts and investments in the last decades to control the epidemic have resulted in a steady decline in disease incidence and mortality [2]. One third of the world population, however, has latent tuberculosis (TB) infection (LTBI), and to reach the United Nations Millennium Goals of eliminating the disease by 2050, it is its necessary to couple diagnosis and treatment of active disease with new approaches to reduce this vast reservoir of LTBI, sufficient for generating new TB cases for many decades even if transmission was suppressed [3]. Thus, in addition to rapid, accurate, and inexpensive detection of active TB, the detection-and treatment-of LTBI is also an important strategy for TB control [1]. In the present paper, we summarize the advantages and limitations of tuberculin skin testing (TST) and overview the evidence for the use of the newer interferon-gamma release assays (IGRA) for the diagnosis of LTBI (Table 1).

\section{Tuberculin Skin Testing}

Until the beginning of this century, TST was the only diagnostic method for detecting LTBI. The test is based on a delayed-type hypersensitivity reaction that occurs when those infected with $M$. tuberculosis are exposed to certain antigenic components present in extracts of culture filtrates, the "tuberculins." In this type of reaction, T cells, sensitized by prior infection, are recruited to the skin where the tuberculin was injected and release lymphokines. The result is local induration of the skin through local vasodilatation, edema, fibrin deposition, and recruitment of other inflammatory cells to the area [4]. An induration greater than $5 \mathrm{~mm}$ is widely accepted as a positive reaction. Different cut-off sizes can be considered. Although widely used, TST has limitations. TST sensitivity may be reduced by malnutrition, severe 
TB diseases and immunodeficiency, such as that related to HIV $[5,6]$. Decreased TST specificity might occur in settings where nontuberculous mycobacteria (NTM) are prevalent and in populations who have received BCG vaccination after infancy, although the effect of BCG vaccination on TST reactions is very modest after 10 years or more if vaccination is given in infancy [7]. Additionally, completing the TST requires two health care visits, for tuberculin injection and induration measurement, which results in loss of reading in approximately $10 \%$ of cases [8]. Also, measurement of reaction size is subject to interobserver variability, although this is greatly reduced with adequate training [9-11]. In addition, one positive TST result does not distinguish recent from remote infection, which has a lower risk of progression to disease $[12,13]$. Although TST reversions are shown to occur, they are more common in older adults, estimated at $8 \%$ per year [14]. When a tuberculin reaction reaches $10 \mathrm{~mm}$ or greater, the additional tests becomes uninterpretable [15], meaning that serial TST has no place in monitoring treatment response. Moreover, repeated injections of tuberculin are known to elicit the booster phenomenon [15]. For this reason, in situations where serial testing is indicated, a two-step tuberculin test is recommended at the time of first testing [16]. Finally, in low- and medium-income countries with a high-TB incidence, diagnosis of LTBI is reported to be difficult because of the extra workload brought by TST [17]. In those countries, detection and treatment of active $\mathrm{TB}$ is the priority, although some could invest in detection and treatment of LTBI if an easier test was available [18].

Yet, TST has many advantages. It is a century-old test that has been widely used, and its clinical applications are very well studied. Cut-off points have been established for indication of isoniazid preventive therapy (IPT) for different ages and risk groups [19]. More importantly, the benefit of IPT in groups with a positive TST has been widely proven, as has been the lack of benefit of IPT in TST-negative subjects $[19,20]$. Finally, there are very clear definitions of conversion and boosting [15]. Conversion is defined as an induration over $10 \mathrm{~mm}$ with an increase of at least $6 \mathrm{~mm}$ over the previous result. This is because the standard deviation of repeated readings (intra- and interobserver variation and within-subject variation) is $3 \mathrm{~mm}$. It is thus expected that $95 \%$ of subjects will have an increase of less than $6 \mathrm{~mm}$ (two standard deviations) not related to TB exposure. In summary, the main application of TST is to detect LTBI, but its role alone for evaluating the risk for progression to disease is limited. It must be interpreted in light of the clinical situation and epidemiological history to allow for evaluation of risk of disease.

\section{Interferon-Gamma Release Assays (IGRAs)}

IGRAs were designed to detect the immune response to specific $M$. tuberculosis antigens, which are not present in BCG or certain nontuberculous mycobacteria. Two tests are commercially available; one is based on the Elispot ( $\mathrm{T}$ SPOT.TB, Oxford Immunotec, UK) and the other on the enzyme-linked immunosorbent assays (ELISA) technique
(QuantiFERON-TB Gold-in-Tube, Cellestis, Australia, QFTGIT). Both tests are based on the principle that the $\mathrm{T}$ cells of an individual who have acquired TB infection will respond by secreting the cytokine interferon-gamma (IFN$\gamma$ ) when restimulated with $M$. tuberculosis antigens [21]. To avoid cross-reactivity, these tests use antigens encoded in the region of difference 1 (RD1), a portion of the Mycobacterium tuberculosis genome that is absent from the genome of BCG and many NTM [21]. The QFT-GIT uses the patient's wholeblood and is based on the ELISA measurement of IFN$\gamma$ produced in response to three $M$. tuberculosis antigens (ESAT-6, CFP-10, and TB7.7). The second test, T-SPOT.TB, is based on the enzyme-linked immunospot measurement of the number of peripheral mononuclear cells that produce IFN- $\gamma$ after stimulation with two mycobacterial antigens: ESAT-6 and CFP-10.

There have been several publications on IGRAs' accuracy, predictive value, cost-effectiveness, and indications since they became available commercially. Many high-income countries have incorporated these tests in their national guidelines [32]. However, the World Health Organization (WHO) has recently published recommendations against their use in low- and medium-income countries (LMIC) [33], since most IGRA studies have been done in highincome countries and mere extrapolation to low- and middleincome settings with high background $\mathrm{TB}$ infection rates being not appropriate. In addition, systematic reviews have suggested that IGRA performance differs in high- versus low-TB and HIV incidence settings, with relatively lower sensitivity in high-burden settings [33]. One other important aspect against their use in LMICs is the cost. Given similar performance but higher costs, IGRAs replacing TSTs as a public health intervention in settings with limited budget being not recommended.

\section{Accuracy of IGRAs to Diagnose LTBI}

The evaluation of the accuracy of these tests to diagnose LTBI has been hampered by the fact that there is no gold standard. Evaluation of new techniques, therefore, relies on the use of proxies for LTBI such as active TB, TB exposure gradients, rates of $\mathrm{TB}$ incidence in untreated cohorts and results of randomized clinical trials comparing the benefit of preventive treatment in patients who test positive and negative. Here, we briefly review the evidence using each of these approaches.

4.1. Active TB. Most of the studies on sensitivity were performed in patients with active $\mathrm{TB}$, relying on the principle that patients with active TB are necessarily infected. The main drawback of this approach is that patients with active TB usually have immunosuppression due to the disease itself or to malnutrition, hampering the ability to respond both to tuberculin and to specific $M$. tuberculosis antigens present in IGRA tests [22]. Thus, sensitivity of both TST and IGRA tests for LTBI is likely to be underestimated in these studies. Conversely, specificity has been evaluated in subjects believed not to have been exposed to TB antigens, which is 
TABLE 1: Comparison of TST and IGRA regarding several tests' characteristics.

\begin{tabular}{|c|c|c|c|}
\hline Test characteristics & TST & QFT & T-SPOT.TB \\
\hline Overall sensitivity [22] & $77 \%$ & $78 \%$ & $92 \%$ \\
\hline HIV uninfected [9] & & $84 \%$ & $88 \%$ \\
\hline HIV infected [18] & & $65 \%$ & $68 \%$ \\
\hline Overall specificity [22] & $97 \%$ & $98 \%$ & $93 \%$ \\
\hline HIV uninfected & & NA & NA \\
\hline HIV infected [18] & & $52 \%$ & $61 \%$ \\
\hline Decreased specificity if BCG vaccinated? & $\begin{array}{l}\text { Yes, particularly if done after } \\
\text { infancy or repeatedly [22] }\end{array}$ & \multicolumn{2}{|c|}{ No effect [22] } \\
\hline Prone to nonspecific variations in test results? & Yes, from reader variability $[10]$ & \multicolumn{2}{|c|}{$\begin{array}{l}\text { Yes, from innumerous factors } \\
\qquad[23-28]\end{array}$} \\
\hline Thresholds for conversions evidence based? & Yes $[15]$ & No & No \\
\hline Prone to conversions? & Yes, mostly due to TB exposure & \multicolumn{2}{|c|}{$\begin{array}{c}\text { Yes (high when only } \\
\text { negative-positive definitions } \\
\text { are used) [29] }\end{array}$} \\
\hline Prone to reversions? & Yes [29] & \multicolumn{2}{|c|}{$\begin{array}{l}\text { High in individuals with weak } \\
\text { IGRA positivity and discordance } \\
\text { with TST [29] }\end{array}$} \\
\hline Boosting? & Yes $[15]$ & \multicolumn{2}{|c|}{$\begin{array}{l}\text { Yes, possibly if given } 3 \text { days } \\
\text { after performing TST [24] }\end{array}$} \\
\hline $\begin{array}{l}\text { Conversion associated with risk of progression to active } \\
\text { TB? }\end{array}$ & Yes (strong evidence) & \multicolumn{2}{|c|}{ No direct evidence } \\
\hline Ability to detect those at high risk of developing active TB & Weak $[30,31]$ & \multicolumn{2}{|c|}{ Weak $[30,31]$} \\
\hline $\begin{array}{l}\text { Preventive treatment of individuals with conversion } \\
\text { reduces risk of progression to active TB? }\end{array}$ & Yes $[19,20]$ & \multicolumn{2}{|c|}{ No evidence } \\
\hline
\end{tabular}

difficult to prove. In a metaanalysis, Pai et al. [22] assessed the accuracy of IGRAs for the diagnosis of LTBI. Sensitivity was determined by using patients with newly diagnosed active TB as a surrogate of latent infection. This meta-analysis included studies performed in low- and high TB-burden countries and found a pooled sensitivity of 78\% (95\% confidence interval (CI), $73 \%$ to $82 \%$ ) for QuantiFERON-TB Gold and $92 \%$ (CI, 90\% to 93\%) for T-SPOT.TB. Sensitivities of both TST and Quanti-FERON were highly heterogeneous, possibly due to HIV coinfection, advanced disease, and malnourishment, which reduce immune response. Indeed, in a study of mostly smear negative active TB patients, that is, with probably early disease, $10 \%$ were TST negative [34], while in a study in Japan, $43 \%$ of patients with advanced TB were TST negative [35]. The specificity studies were all from countries with low rate of TB incidence with varying BCG policies and found a pooled specificity of 98\% (CI, 96\% to 99\%) for all QFT studies, with little difference among BCG and non-BCGvaccinated populations (96\% versus 99\%, resp.) and 93\% (CI, $86 \%$ to $100 \%$ ) for T-SPOT.TB (only one study among BCGvaccinated subjects). The TST sensitivity was similar, with a pooled estimate of $77 \%$ (CI, $71 \%$ to $82 \%$ ). Specificity, however, showed a more marked difference between non-BCG and BCG-vaccinated participants. In non-BCG-vaccinated subjects, the pooled estimate was consistently high, 97\% (CI, $95 \%$ to $99 \%$ ) but in the BCG vaccinated population specificity was only $59 \%$ (CI, $46 \%$ to $73 \%$ ). Four of the 6 studies, however, were from countries where BCG vaccination is done after infancy or done multiple times [36]. No studies have directly compared TST and IGRAs in populations that were BCG vaccinated only at birth, where specificity of TST is much higher.

4.2. Exposure Gradients. Usually, TB exposure is captured either as a dichotomous variable (exposed and unexposed) or as a gradient (degree of exposure is graded according to duration, or proximity). In exposure gradient studies, contacts are assigned to 3 or more categories from most to least exposed. The proportion of positive results should parallel the exposure gradient. The best test should demonstrate the closest correlation of proportion positive to this gradient. The main challenge with these methods is the difficulty in quantifying the exact degree of exposure of contacts. In addition, sometimes the criteria for exposure are different among studies, making pooled estimates difficult. A total of 10 studies in pediatric populations used this method [37-46]. There was great variation in the definition of the exposure groups. For TST, most studies used a $10 \mathrm{~mm}$ cutoff point, while 2 studies used $5 \mathrm{~mm}$ and one study $15 \mathrm{~mm}$. The overall pooled odds ratio (OR) was 1.34 (CI, 0.66-2.72), 1.93 (CI, 0.98-3.77), and 1.83 (CI, 0.67-5.02) for TST when 
$5 \mathrm{~mm}, 10 \mathrm{~mm}$, or $15 \mathrm{~mm}$ were used, respectively, as the cutoff value [47]. For QuantiFERON, the pooled OR was 3.51 (CI, 1.85-6.66), and for T-SPOT it was 1.31 (CI, 0.76-2.27) [47]. All confidence intervals overlapped, indicating similar performance for all tests [47].

4.3. Incidence of TB in Cohorts. More important than identifying individuals with LTBI is the recognition of those who will progress to active disease, because those are the subjects who will benefit from isoniazid preventive therapy (IPT). So far, IGRAs have been shown to have a low predictive ability for progression from LTBI to active disease although individuals with positive IGRA have slightly higher risk than those with positive TST results [30, 48-51].

Two recent meta-analyses have reviewed the predictive value of TST and QFT [30, 31]. One review included fifteen studies comparing the incidence of active TB in IGRApositive and TST-positive subjects [31], including studies in children, healthcare workers, and PLWHA [47, 52, 53]. Among these studies, 5 looked at the risk for progression to TB in PLWHA [54-58]. Three showed a higher risk in IGRApositive compared to IGRA-negative subjects [54-56], but 2 did not $[57,58]$. By contrast, the risk of developing active TB in TST-positive PLWHA is well documented. Moreover, the benefit of IPT in TST-positive PLWHA has been confirmed in many studies $[20,59]$. Overall, the incidence rate of TB over a median time of 4 years was very low in both TST- and IGRA-positive test subjects (3.7 to 4.8 and 1.0 to 4.5 per 1000 persons-year, resp.). These low incidence rates show that both tests have a low predictive value for active disease.

The second review on the predictive value of IGRAs [30] included more studies, since it did not exclude those in which IGRA results were not blinded to the treating physician, ignoring the risk of "incorporation bias" of these studies. Although the authors found that progression rates for commercial IGRAs were significantly higher than those for the TST $(2.7 \%$ versus $1.5 \%)$, they also concluded that they are both low in absolute numbers [30].

\subsection{Evidence of Benefit from Isoniazid Preventive Therapy.} More important than establishing the accuracy and predictive value of these tests is to evaluate the benefit from IPT in reducing the risk for progression to active disease in subjects with a positive result. While there is very strong evidence of the protection from IPT in TST-positive subjects [19, 20], so far there are no studies showing direct evidence of the efficacy of IPT based on IGRA results. Indirect evidence of this benefit is based on isoniazid protection against TB in patients who have a positive TST. A recent study in Botswana has shown that long-term IPT in TST-positive persons living with HIV/AIDS (PLWHA) has a strong impact on survival. The study has also shown that isoniazid has little benefit in PLWHA TST-negative individuals [20,59].

TST conversion usually reflects recent infection. The risk of progression to disease in recent converters is still higher, thus, IPT is recommended, regardless of HIV status or background of TB incidence. However, for PLWHA who become TST positive after initiating highly active antiretroviral therapy (HAART), conversion should be interpreted with caution, as it may be due to immune reconstitution and not recent infection.

Given the similarity of TST and IGRAs in cohort studies, it is reasonable to believe that IPT in IGRA-positive individuals should provide at least the same benefit as in TST-positive individuals [60], but the lack of benefit in IGRA-negative individuals is yet to be evaluated.

\section{IGRA as a Marker of Cure Following Treatment of LTBI}

As previously remarked, TST reversion is not associated with successful treatment of LTBI. To test the usefulness of IGRAs to monitor TB and LTBI treatment, IGRA tests have been applied serially during and after treatment of patients with active TB and of healthy subjects undergoing LTBI treatment [61-76]. Overall, results were highly contradictory, with increases or decreases of interferon-gamma levels being observed randomly, regardless of clinical response to treatment [77]. Chee et al. [63] compared QFT-GIT and the TSPOT.TB at baseline, after treatment completion and after 6 months in sputum-positive, immunocompetent patients. Although there was a significant rate of reversions $(13.9 \%$ for T-SPOT.TB versus $39.2 \%$ for QFT-IT), a substantial proportion of patients remained test positive 6 months after TB treatment (79\% and 46\% with T-SPOT.TB and QFT-GIT, resp.). There are no long-term studies of IGRA in the followup of subjects treated for LTBI, but a few short-term studies have shown that a decrease in quantitative results can occur after IPT, regardless of reversion. Dyrhol-Riise et al. [71] tested the performance of QFT-GIT for monitoring LTBI treatment in patients before and three and 15 months after the beginning of isoniazid and rifampicin treatment. The IFN- $\gamma$ responses were comparable at the three time points, with no statistically significant change during the course of treatment. A recent meta-analysis supports these findings [77]. In summary, IGRAs have, at present, no place in monitoring response to treatment of TB or LTBI.

\section{LTBI in Special Populations}

The accuracy of IGRA tests have been tested in distinct subpopulations such as children [38, 47, 48, 56, 78-84], PLWHA $[23,29,39,40,47,54,55,85-103]$, other immunosuppressed patients (those with renal disease or in use of tumor necrosis factor-alpha inhibitors), [104-106] and healthcare workers [53].

6.1. Immunosuppressed Patients. As in immunocompetent populations, the sensitivity of IGRA tests in PLWHA has also been analyzed in patients with active TB. In 18 studies, 16 of them in LMIC [52], sensitivities of both TST and IGRA tests were lower in PLWHA because both require an adequate immune response. Pooled sensitivity of QFT-GIT was $60 \%$ (CI, 34 to $82 \%)$. T-SPOT.TB seemed slightly less affected by immunosuppression, with a pooled sensitivity of $76 \%$ 
(CI, $45 \%$ to $92 \%$ ) [18], but only five studies compared their sensitivity head to head $[93,107-110]$, with only one study showing QFT-GIT with a greater sensitivity than T-SPOT.TB in PLWHA [110]. Likewise, 14 studies have analyzed the role of IGRA in patients with autoimmune disease [111-124], 10 of them in countries where BCG vaccination is not routinely done [111-113, 116-118, 120, 121, 123, 124]. Most studies included a small number of patients, immunosuppressive therapy was variable or not yet started, and very few were conducted in countries with high-TB prevalence. Above all, no longitudinal study to assess the risk of progression to disease was carried out in this population [106]. Finally, there are no studies on the role of IGRA tests among transplanted patients under immunosuppressive therapy.

As mentioned above, special caution is needed when interpreting conversion and reversions in PLWHA. Reversion can be a consequence of declining immunity and should alert the physician. In contrast, LTBI/TB treatment of naive TSTnegative individuals with advanced HIV infection (CD4+ cell count $<200 / \mu \mathrm{L}$ ) started on HAART should be retested for LTBI once they achieve CD4+ cell counts $>200 / \mu \mathrm{L}$ [125].

6.2. Children. Regarding LTBI in the youngest, although a few studies have shown a lower sensitivity of IGRA tests in very young children [39, 40,47,87, 126-128], the sensitivity and specificity of IGRA in older children exposed to index cases are similar than in adults, as long as they are not infected by HIV [47]. Although BCG is not expected to interfere with IGRAs, the lower sensitivity found both in TST and IGRAs among very young children might be explained by immune immaturity or by capturing a population with underlying conditions that may interfere with immunity, such as coinfections with helminths and malnutrition [129]. Overall, the sensitivity and specificity among all children with active TB were similar for TST, QFT-G/QFT-GIT (QFT), and T-SPOT.TB, with a pooled sensitivity of $80 \%$ (CI, 70\% to $90 \%$ ), $83 \%$ (CI, $75 \%$ to $92 \%$ ), and $84 \%$ (63\% to $100 \%$ ), respectively. Pooled specificity for TST, QFT, and T-SPOT.TB was $85 \%$ (CI, $63 \%$ to $100 \%$ ), $91 \%$ (CI, $78 \%$ to $100 \%$ ), and $94 \%$ (87\% to $100 \%)$, respectively [47].

\subsection{Highly Exposed Populations and Serial Testing. Highly} exposed groups, such as health care workers and prisoners, as well as patients at high risk for disease, such as PLWHA, are screened for LTBI every 6 to 12 months. Although it has been described that tuberculin injection may also result in boosting of IGRA responses, this effect is not seen if QFTGIT or T-SPOT.TB is done within 3 days of performing the TST $[24,130]$.

Unlike TST, which should only be repeated if previously negative, IGRA tests may be repeated, regardless of their previous results. However, serial testing subjects not undergoing treatment indicate that there are high rates of spontaneous reversions and conversions, although it is always harder to know if conversion is spontaneous or a consequence of real TB infections [23, 131-135]. Other possible explanations for a conversion would be nonspecific infections and vaccines, laboratorial technique variability, such as blood withdrawal preparation, delay to and time of incubation, and lot of the kit. Biological variables might also interfere. Although there is some evidence that QFT-GIT conversion is related to a greater risk of progression to TB [136], the predictive value of IGRA (QFT) conversion for the development of TB disease is still controversial, and fluctuations in IFN- $\gamma$ responses among serially tested individuals reported in longitudinal studies remain unexplained and nonspecific.

In addition, spontaneous reversions are more likely to occur in subjects with borderline results, close to the cutoff value, are not related to treatment, and are more likely if TST negative [24, 137-140]. In such cases, it has been interpreted as self-clearance of infection, but there is not enough evidence for such a conclusion [71, 125, 141]. For these reasons, serial testing with QFT-GIT and T.SPOT-TB should be considered unreliable and is not recommended at least until the conversion and reversion phenomena are better understood.

\section{Conclusions and Recommendations}

There is still a lot to be learned about IGRAs. Until then, IGRAs can be interpreted similarly to TST for the diagnosis of LTBI; with the exception of populations BCG vaccinated after infancy, IGRAs have a better positive predictive value because of their higher specificity.

More importantly, although both TST and IGRAs are useful for detecting LTBI, neither test is all that accurate for predicting risk for disease alone, and positive tests must be interpreted in light of other clinical risk factors. LTBI testing screening is recommended only for those with a known risk factor for progression to disease, such as recent infection, young age, and immune suppression. Clinicians can be confronted with discordant TST and IGRA results and be unsure of how to manage these patients. Because protection from IPT is well established only among TST+ subjects, and because spontaneous reversion of positive IGRA is very common if the TST is negative, the authors recommend not treating TST-/IGRA+ individuals, unless they have very high risk for disease, as in HIV-positive individuals, and are clearly exposed to TB, in which case treatment for LTBI should be considered [142].

Physicians still need to rely on clinical and epidemiological grounds in order to decide if IPT is indicated in an individual patient with a positive TST or IGRA. This decision should take into account the presence of risk factors for development of disease and the risk for hepatotoxicity from IPT, which increases with age. Patients' preferences should also be considered. To help physicians decide, an interesting tool has been developed and is accessible at http://www.tstin3d.com [12].

As for treatment monitoring and other uses of serial testing, more knowledge is needed before we can incorporate IGRAs in clinical practice.

\section{Acknowledgments}

This review was supported by $\mathrm{CNPq}$ (558383/2009-2), FAPERJ (E-26/102.712/2008 and E-26/110.637/2012), and 
ICOHRTA (5U 2R TW006883-02). None of the funding agencies is responsible for the statements in this paper. The authors declare no conflict of interests.

\section{References}

[1] World Health Organization, Global Tuberculosis Control: WHO Report 2011, World Health Organization, Geneva, Switzerland, 2011.

[2] C. Dye, C. J. Watt, D. M. Bleed, S. M. Hosseini, and M. C. Raviglione, "Evolution of tuberculosis control and prospects for reducing tuberculosis incidence, prevalence, and deaths globally," Journal of the American Medical Association, vol. 293, no. 22, pp. 2767-2775, 2005.

[3] C. Dye and B. G. Williams, "Eliminating human tuberculosis in the twenty-first century," Journal of the Royal Society Interface, vol. 5, no. 23, pp. 653-662, 2008.

[4] R. E. Huebner, M. F. Schein, and J. B. Bass, "The tuberculin skin test," Clinical Infectious Diseases, vol. 17, no. 6, pp. 968-975, 1993.

[5] E. L. Pesanti, "The negative tuberculin test: tuberculin, HIV, and anergy panels," American Journal of Respiratory and Critical Care Medicine, vol. 149, no. 6, pp. 1699-1709, 1994.

[6] R. E. Huebner, M. E. Villarino, and D. E. Snider, "Tuberculin skin testing and the HIV epidemic," Journal of the American Medical Association, vol. 267, no. 3, pp. 409-410, 1992.

[7] M. Farhat, C. Greenaway, M. Pai, and D. Menzies, "Falsepositive tuberculin skin tests: what is the absolute effect of BCG and non-tuberculous mycobacteria?" International Journal of Tuberculosis and Lung Disease, vol. 10, no. 11, pp. 1192-1204, 2006.

[8] R. Diel, A. Nienhaus, C. Lange, and T. Schaberg, "Costoptimisation of screening for latent tuberculosis in close contacts," European Respiratory Journal, vol. 28, no. 1, pp. 35-44, 2006.

[9] J. E. Bearman, H. Kleinman, V. V. Glyer, and O. M. Lacroix, "A study of variability in tuberculin test reading," American Review of Respiratory Disease, vol. 90, pp. 913-919, 1964.

[10] S. D. Chaparas, H. M. Vandiviere, and I. Melvin, "Tuberculin test. Variability with the Mantoux procedure," American Review of Respiratory Disease, vol. 132, no. 1, pp. 175-177, 1985.

[11] E. Perez-Stable and G. Slutkin, "A demonstration of lack of variability among six tuberculin skin test readers," American Journal of Public Health, vol. 75, no. 11, pp. 1341-1343, 1985.

[12] D. Menzies, G. Gardiner, M. Farhat, C. Greenaway, and M. Pai, "Thinking in three dimensions: a web-based algorithm to aid the interpretation of tuberculin skin test results," International Journal of Tuberculosis and Lung Disease, vol. 12, no. 5, pp. 498-505, 2008.

[13] E. Vynnycky and P. E. M. Fine, "The natural history of tuberculosis: the implications of age-dependent risks of disease and the role of reinfection," Epidemiology and Infection, vol. 119, no. 2, pp. 183-201, 1997.

[14] S. Grzybowski and E. A. Allen, "The challenge of tuberculosis in decline. A study based on the epidemiology of tuberculosis in ontario, Canada," The American Review of Respiratory Disease, vol. 90, pp. 707-720, 1964.

[15] D. Menzies, "Interpretation of repeated tuberculin tests: boosting, conversion, and reversion," American Journal of Respiratory and Critical Care Medicine, vol. 159, no. 1, pp. 15-21, 1999.
[16] J. B. Bass Jr. and R. A. Serio, "The use of repeat skin tests to eliminate the booster phenomenon in serial tuberculin testing," American Review of Respiratory Disease, vol. 123, no. 4, pp. 394-396, 1981.

[17] D. Menzies, H. Al Jahdali, and B. Al Otaibi, "Recent developments in treatment of latent tuberculosis infection," Indian Journal of Medical Research, vol. 133, no. 3, pp. 257-266, 2011.

[18] J. Z. Metcalfe, C. K. Everett, K. R. Steingart, A. Cattamanchi, L. Huang, P. C. Hopewell et al., "Interferon- $\gamma$ release assays for active pulmonary tuberculosis diagnosis in adults in lowand middle-income countries: systematic review and metaanalysis," Journal of Infectious Diseases, vol. 204, supplement 4, pp. S1120-S1129, 2011.

[19] H. C. Bucher, L. E. Griffith, G. H. Guyatt et al., "Isoniazid prophylaxis for tuberculosis in HIV infection: a meta-analysis of randomized controlled trials," AIDS, vol. 13, no. 4, pp. 501-507, 1999.

[20] T. Samandari, T. B. Agizew, S. Nyirenda et al., "6-month versus 36-month isoniazid preventive treatment for tuberculosis in adults with HIV infection in Botswana: a randomised, doubleblind, placebo-controlled trial," The Lancet, vol. 377, no. 9777, pp. 1588-1598, 2011.

[21] P. Andersen, M. E. Munk, J. M. Pollock, and T. M. Doherty, "Specific immune-based diagnosis of tuberculosis," The Lancet, vol. 356, no. 9235, pp. 1099-1104, 2000.

[22] M. Pai, A. Zwerling, and D. Menzies, "Systematic review: T-cellbased assays for the diagnosis of latent tuberculosis infection: an update," Annals of Internal Medicine, vol. 149, no. 3, pp. 177-184, 2008.

[23] M. Pai, R. Joshi, S. Dogra et al., “T-cell assay conversions and reversions among household contacts of tuberculosis patients in rural India," International Journal of Tuberculosis and Lung Disease, vol. 13, no. 1, pp. 84-92, 2009.

[24] K. Dheda, R. N. Van Zyl-Smit, L. A. Sechi et al., "Utility of quantitative T-cell responses versus unstimulated interferon- $\gamma$ for the diagnosis of pleural tuberculosis," European Respiratory Journal, vol. 34, no. 5, pp. 1118-1126, 2009.

[25] A. K. Detjen, L. Loebenberg, H. M. S. Grewal et al., "Shortterm reproducibility of a commercial interferon gamma release assay," Clinical and Vaccine Immunology, vol. 16, no. 8, pp. 1170-1175, 2009.

[26] D. Doberne, R. L. Gaur, and N. Banaei, "Preanalytical delay reduces sensitivity of QuantiFERON-TB gold in-tube assay for detection of latent tuberculosis infection," Journal of Clinical Microbiology, vol. 49, no. 8, pp. 3061-3064, 2011.

[27] F. C. Ringshausen, A. Nienhaus, J. Torres Costa, H. Knoop, S. Schlosser, G. Schultze-Werninghaus et al., "Within-subject variability of Mycobacterium tuberculosis-specific gamma interferon responses in German health care workers," Clinical and Vaccine Immunology, vol. 18, no. 7, pp. 1176-1182, 2011.

[28] M. Slater, J. Parsonnet, and N. Banaei, "Investigation of falsepositive results given by the QuantiFERON-TB gold in-tube assay," Journal of Clinical Microbiology, vol. 50, no. 9, pp. 3105-3107, 2012.

[29] M. Pai and R. O'Brien, "Serial testing for tuberculosis: can we make sense of T cell assay conversions and reversions?" PLoS Medicine, vol. 4, no. 6, Article ID e208, 2007.

[30] R. Diel, R. Loddenkemper, and A. Nienhaus, "Predictive value of interferon-gamma release assays and tuberculin skin testing for predicting progression from latent TB infection to disease state: a meta-analysis," Chest, vol. 142, no. 1, pp. 63-75, 2012. 
[31] M. X. Rangaka, K. A. Wilkinson, J. R. Glynn, D. Ling, D. Menzies, J. Mwansa-Kambafwile et al., "Predictive value of interferon-gamma release assays for incident active tuberculosis: a systematic review and meta-analysis," The Lancet Infectious Diseases, vol. 12, no. 1, pp. 45-55, 2012.

[32] C. M. Denkinger, K. Dheda, and M. Pai, "Guidelines on interferon- $\gamma$ release assays for tuberculosis infection: concordance, discordance or confusion?" Clinical Microbiology and Infection, vol. 17, no. 6, pp. 806-814, 2011.

[33] World Health Organization, Use of Tuberculosis InterferonGamma Release Assays (IGRAs) in Low- and Middle-Income Countries: Policy Statement, World Health Organization, Geneva, Switzerland, 2011.

[34] S. J. Tsiouris, D. Coetzee, P. L. Toro, J. Austin, Z. Stein, and W. ElSadr, "Sensitivity analysis and potential uses of a novel gamma interferon release assay for diagnosis of tuberculosis," Journal of Clinical Microbiology, vol. 44, no. 8, pp. 2844-2850, 2006.

[35] Y. Kobashi, K. Mouri, S. Yagi et al., "Usefulness of the QuantiFERON TB-2G test for the differential diagnosis of pulmonary tuberculosis," Internal Medicine, vol. 47, no. 4, pp. 237-243, 2008.

[36] A. Zwerling, M. A. Behr, A. Verma, T. F. Brewer, D. Menzies, and M. Pai, "The BCG world atlas: a database of global BCG vaccination policies and practices," PLoS Medicine, vol. 8, no. 3, Article ID e1001012, 2011.

[37] J. K. Chun, C. K. Kim, H. S. Kim et al., "The role of a whole blood interferon- $\gamma$ assay for the detection of latent tuberculosis infection in Bacille Calmette-Guérin vaccinated children," Diagnostic Microbiology and Infectious Disease, vol. 62, no. 4, pp. 389-394, 2008.

[38] L. Bianchi, L. Galli, M. Moriondo et al., "Interferon-gamma release assay improves the diagnosis of tuberculosis in children," Pediatric Infectious Disease Journal, vol. 28, no. 6, pp. 510-514, 2009.

[39] E. Hansted, A. Andriuskeviciene, R. Sakalauskas, R. Kevalas, and B. Sitkauskiene, "T-cell-based diagnosis of tuberculosis infection in children in Lithuania: a country of high incidence despite a high coverage with bacille Calmette-Guerin vaccination," BMC Pulmonary Medicine, vol. 9, article 41, 2009.

[40] A. C. Hesseling, A. M. Mandalakas, H. L. Kirchner et al., "Highly discordant $\mathrm{T}$ cell responses in individuals with recent exposure to household tuberculosis," Thorax, vol. 64, no. 10, pp. 840-846, 2009.

[41] K. Higuchi, Y. Kawabe, S. Mitarai, T. Yoshiyama, N. Harada, and T. Mori, "Comparison of performance in two diagnostic methods for tuberculosis infection," Medical Microbiology and Immunology, vol. 198, no. 1, pp. 33-37, 2009.

[42] J. Lighter, M. Rigaud, R. Eduardo, C. H. Peng, and H. Pollack, "Latent tuberculosis diagnosis in children by using the QuantiFERON-TB gold in-tube test," Pediatrics, vol. 123, no. 1, pp. 30-37, 2009.

[43] M. Lucas, P. Nicol, E. McKinnon et al., "A prospective largescale study of methods for the detection of latent Mycobacterium tuberculosis infection in refugee children," Thorax, vol. 65, no. 5, pp. 442-448, 2010.

[44] D. C. Stefan, A. Dippenaar, A. K. Detjen et al., "Interferongamma release assays for the detection of Mycobacterium tuberculosis infection in children with cancer," International Journal of Tuberculosis and Lung Disease, vol. 14, no. 6, pp. 689-694, 2010.
[45] A. M. Mandalakas, A. C. Hesseling, N. N. Chegou et al., "High level of discordant IGRA results in HIV-infected adults and children," International Journal of Tuberculosis and Lung Disease, vol. 12, no. 4, pp. 417-423, 2008.

[46] J. Domínguez, J. Ruiz-Manzano, M. De Souza-Galvão et al., "Comparison of two commercially available gamma interferon blood tests for immunodiagnosis of tuberculosis," Clinical and Vaccine Immunology, vol. 15, no. 1, pp. 168-171, 2008.

[47] A. M. Mandalakas, A. K. Detjen, A. C. Hesseling, A. Benedetti, and D. Menzies, "Interferon-gamma release assays and childhood tuberculosis: systematic review and meta-analysis," International Journal of Tuberculosis and Lung Disease, vol. 15, no. 8, pp. 1018-1032, 2011.

[48] M. Bakir, K. A. Millington, A. Soysal et al., "Prognostic value of a T-cell-based, interferon- $\gamma$ biomarker in children with tuberculosis contact," Annals of Internal Medicine, vol. 149, no. 11, pp. 777-786, 2008.

[49] H. del Corral, S. C. Paris, N. D. Marin, D. M. Marin, L. Lopez, H. M. Henao et al., "IFNgamma response to Mycobacterium tuberculosis, risk of infection and disease in household contacts of tuberculosis patients in Colombia," PLoS ONE, vol. 4, no. 12, Article ID e8257, 2009.

[50] P. C. Hill, D. J. Jackson-Sillah, A. Fox et al., "Incidence of tuberculosis and the predictive value of ELISPOT and Mantoux tests in Gambian case contacts," PLoS ONE, vol. 3, no. 1, Article ID e1379, 2008.

[51] S. V. Kik, W. P. J. Franken, M. Mensen et al., "Predictive value for progression to tuberculosis by IGRA and TST in immigrant contacts," European Respiratory Journal, vol. 35, no. 6, pp. 1346-1353, 2010.

[52] A. Cattamanchi, R. Smith, K. R. Steingart et al., "Interferongamma release assays for the diagnosis of latent tuberculosis infection in HIV-infected individuals: a systematic review and meta-analysis," Journal of Acquired Immune Deficiency Syndromes, vol. 56, no. 3, pp. 230-238, 2011.

[53] A. Zwerling, S. van den Hof, J. Scholten, F. Cobelens, D. Menzies, and M. Pai, "Interferon-gamma release assays for tuberculosis screening of healthcare workers: a systematic review," Thorax, vol. 67, no. 1, pp. 62-70, 2011.

[54] M. C. Aichelburg, A. Rieger, F. Breitenecker et al., "Detection and prediction of active tuberculosis disease by a wholeblood interferon- $\gamma$ release assay in HIV-l-infected individuals," Clinical Infectious Diseases, vol. 48, no. 7, pp. 954-962, 2009.

[55] S. A. Clark, S. L. Martin, A. Pozniak et al., "Tuberculosis antigen-specific immune responses can be detected using enzyme-linked immunospot technology in human immunodeficiency virus (HIV)-1 patients with advanced disease," Clinical and Experimental Immunology, vol. 150, no. 2, pp. 238-244, 2007.

[56] S. Jonnalagadda, B. L. Payne, E. Brown et al., "Latent tuberculosis detection by interferon $\gamma$ release assay during pregnancy predicts active tuberculosis and mortality in human immunodeficiency virus type 1-infected women and their children," Journal of Infectious Diseases, vol. 202, no. 12, pp. 1826-1835, 2010.

[57] M. Santin, S. Casas, M. Saumoy et al., "Detection of latent tuberculosis by the tuberculin skin test and a whole-blood interferon- $\gamma$ release assay, and the development of active tuberculosis in HIV-seropositive persons," Diagnostic Microbiology and Infectious Disease, vol. 69, no. 1, pp. 59-65, 2011. 
[58] L. F. Zhang, X. Q. Liu, L. Y. Zuo, T. S. Li, G. H. Deng, and A. $\mathrm{X}$. Wang, "Longitudinal observation of an interferon gammareleased assay (T-SPOT.TB) for Mycobacterium tuberculosis infection in AIDS patients on highly active antiretroviral therapy," Chinese Medical Journal, vol. 123, no. 9, pp. 1117-1121, 2010.

[59] C. Akolo, I. Adetifa, S. Shepperd, and J. Volmink, "Treatment of latent tuberculosis infection in HIV infected persons," Cochrane Database of Systematic Reviews, no. 1, Article ID CD000171, 2010.

[60] D. W. Dowdy and J. E. Golub, "Tests for latent tuberculosis infection and isoniazid preventive therapy," The Lancet Infectious Diseases, vol. 12, no. 11, pp. 827-828, 2012.

[61] S. Ribeiro, K. Dooley, J. Hackman et al., “T-SPOT.TB responses during treatment of pulmonary tuberculosis," BMC Infectious Diseases, vol. 9, article 23, 2009.

[62] V. Bosshard, P. Roux-Lombard, T. Perneger et al., "Do results of the T-SPOT.TB interferon- $\gamma$ release assay change after treatment of tuberculosis?" Respiratory Medicine, vol. 103, no. 1, pp. 30-34, 2009.

[63] C. B. Chee, K. W. KhinMar, S. H. Gan, T. M. Barkham, C. K. Koh, L. Shen et al., "Tuberculosis treatment effect on T-cell interferon-gamma responses to Mycobacterium tuberculosisspecific antigens," European Respiratory Journal, vol. 36, no. 2, pp. 355-361, 2010.

[64] I. Latorre, N. Altet, M. de Souza-Galvao, J. Ruiz-Manzano, A. Lacoma, C. Prat et al., "Specific Mycobacterium tuberculosis T cell responses to RD1-selected peptides for the monitoring of anti-tuberculosis therapy," Scandinavian Journal of Infectious Diseases, vol. 44, no. 3, pp. 161-167, 2012.

[65] J. Domínguez, M. D. Souza-Galvão, J. Ruiz-Manzano et al., "T-cell responses to the Mycobacterium tuberculosis-specific antigens in active tuberculosis patients at the beginning, during, and after antituberculosis treatment," Diagnostic Microbiology and Infectious Disease, vol. 63, no. 1, pp. 43-51, 2009.

[66] K. Dheda, A. Pooran, M. Pai et al., "Interpretation of Mycobacterium tuberculosis antigen-specific IFN- $\gamma$ release assays (TSPOT.TB) and factors that may modulate test results," Journal of Infection, vol. 55, no. 2, pp. 169-173, 2007.

[67] W. P. J. Franken, S. M. Arend, S. F. T. Thijsen et al., "Interferongamma release assays during follow-up of tuberculin skin testpositive contacts," International Journal of Tuberculosis and Lung Disease, vol. 12, no. 11, pp. 1286-1294, 2008.

[68] I. Sauzullo, F. Mengoni, M. Lichtner et al., "In vivo and in vitro effects of antituberculosis treatment on mycobacterial interferon- $\gamma$ T cell response," PLoS ONE, vol. 4, no. 4, Article ID e5187, 2009.

[69] Y. Kobashi, T. Sugiu, Y. Ohue et al., "Long-term follow-up of the QuantiFERON TB-2G test for active tuberculosis disease," Internal Medicine, vol. 47, no. 22, pp. 1957-1961, 2008.

[70] D. Goletti, M. P. Parracino, O. Butera et al., "Isoniazid prophylaxis differently modulates $\mathrm{T}$-cell responses to RD1-epitopes in contacts recently exposed to Mycobacterium tuberculosis: a pilot study," Respiratory Research, vol. 8, article 5, 2007.

[71] A. M. Dyrhol-Riise, G. Gran, T. Wenzel-Larsen, B. Blomberg, C. G. Haanshuus, and O. Mørkve, "Diagnosis and followup of treatment of latent tuberculosis; the utility of the QuantiFERON-TB Gold In-tube assay in outpatients from a tuberculosis low-endemic country," BMC Infectious Diseases, vol. 10, article 57, 2010.
[72] S. H. Lee, W. J. Lew, H. J. Kim et al., "Serial interferon-gamma release assays after rifampicin prophylaxis in a tuberculosis outbreak," Respiratory Medicine, vol. 104, no. 3, pp. 448-453, 2010.

[73] S. W. Lee, C. T. Lee, and J. J. Yim, "Serial interferon-gamma release assays during treatment of active tuberculosis in young adults," BMC Infectious Diseases, vol. 10, article 300, 2010.

[74] M. Bocchino, P. Chairadonna, A. Matarese et al., "Limited usefulness of QuantiFERON-TB GOLD in-tube for monitoring anti-tuberculosis therapy," Respiratory Medicine, vol. 104, no. 10, pp. 1551-1556, 2010.

[75] J. L. Herrmann, M. Belloy, R. Porcher et al., "Temporal dynamics of interferon gamma responses in children evaluated for tuberculosis," PLoS ONE, vol. 4, no. 1, Article ID e4130, 2009.

[76] N. Nenadic, B. K. Kirin, I. Z. Letoja, D. Plavec, R. Z. Topic, and S. Dodig, "Serial interferon-gamma release assay in children with latent tuberculosis infection and children with tuberculosis," Pediatric Pulmonology, vol. 47, no. 4, pp. 401-408, 2012.

[77] E. Chiappini, F. Fossi, F. Bonsignori, S. Sollai, L. Galli, and M. de Martino, "Utility of interferon-gamma release assay results to monitor anti-tubercular treatment in adults and children," Clinical Therapeutics, vol. 34, no. 5, pp. 1041-1048, 2012.

[78] A. R. J. Bamford, A. M. Crook, J. E. Clark et al., "Comparison of interferon- $\gamma$ release assays and tuberculin skin test in predicting active tuberculosis (TB) in children in the UK: a paediatric TB network study," Archives of Disease in Childhood, vol. 95, no. 3, pp. 180-186, 2010.

[79] T. G. Connell, M. A. Davies, C. Johannisen et al., "Reversion and conversion of Mycobacterium tuberculosis IFN- $\gamma$ ELISpot results during anti-tuberculous treatment in HIV-infected children," BMC Infectious Diseases, vol. 10, article 138, 2010.

[80] B. Kampmann, E. Whittaker, A. Williams et al., "Interferon$\gamma$ release assays do not identify more children with active tuberculosis than the tuberculin skin test," European Respiratory Journal, vol. 33, no. 6, pp. 1374-1382, 2009.

[81] D. A. Lewinsohn, M. N. Lobato, and J. A. Jereb, "Interferon- $\gamma$ release assays: new diagnostic tests for Mycobacterium tuberculosis infection, and their use in children," Current Opinion in Pediatrics, vol. 22, no. 1, pp. 71-76, 2010.

[82] S. MacHingaidze, C. S. Wiysonge, Y. Gonzalez-Angulo et al., "The utility of an interferon gamma release assay for diagnosis of latent tuberculosis infection and disease in children: a systematic review and meta-analysis," Pediatric Infectious Disease Journal, vol. 30, no. 8, pp. 694-700, 2011.

[83] N. Nenadic, B. K. Kirin, I. Z. Letoja, D. Plavec, R. Z. Topic, and S. Dodig, "Serial interferon- $\gamma$ release assay in children with latent tuberculosis infection and children with tuberculosis," Pediatric Pulmonology, vol. 47, no. 4, pp. 401-408, 2012.

[84] M. P. Nicol, M. A. Davies, K. Wood et al., "Comparison of TSPOT. TB assay and tuberculin skin test for the evaluation of young children at high risk for tuberculosis in a community setting," Pediatrics, vol. 123, no. 1, pp. 38-43, 2009.

[85] M. G. Aabye, P. Ravn, G. PrayGod et al., "The impact of HIV infection and CD4 cell count on the performance of an interferon gamma release assay in patients with pulmonary tuberculosis," PLoS ONE, vol. 4, no. 1, Article ID e4220, 2009.

[86] I. M. O. Adetifa, M. D. Lugos, A. Hammond et al., "Comparison of two interferon gamma release assays in the diagnosis of Mycobacterium tuberculosis infection and disease in The Gambia," BMC Infectious Diseases, vol. 7, article 122, 2007. 
[87] I. M. O. Adetifa, M. O. C. Ota, D. J. Jeffries et al., "Commercial interferon gamma release assays compared to the tuberculin skin test for diagnosis of latent Mycobacterium tuberculosis infection in childhood contacts in the gambia," Pediatric Infectious Disease Journal, vol. 29, no. 5, pp. 439-443, 2010.

[88] K. Baba, S. Sørnes, A. A. Hoosen et al., "Evaluation of immune responses in HIV infected patients with pleural tuberculosis by the QuantiFERON TB-Gold interferon-gamma assay," BMC Infectious Diseases, vol. 8, article 35, 2008.

[89] I. Brock, M. Ruhwald, B. Lundgren, H. Westh, L. R. Mathiesen, and P. Ravn, "Latent tuberculosis in HIV positive, diagnosed by the M. tuberculosis specific interferon- $\gamma$ test," Respiratory Research, vol. 7, article 56, 2006.

[90] A. Cattamanchi, I. Ssewenyana, J. L. Davis et al., "Role of interferon-gamma release assays in the diagnosis of pulmonary tuberculosis in patients with advanced HIV infection," $B M C$ Infectious Diseases, vol. 10, article 75, 2010.

[91] K. Dheda, A. Lalvani, R. F. Miller et al., "Performance of a T-cellbased diagnostic test for tuberculosis infection in HIV-infected individuals is independent of CD4 cell count," AIDS, vol. 19, no. 17, pp. 2038-2041, 2005.

[92] K. Dheda, R. Van Zyl Smit, M. Badri, and M. Pai, "T-cell interferon- $\gamma$ release assays for the rapid immunodiagnosis of tuberculosis: clinical utility in high-burden vs. low-burden settings," Current Opinion in Pulmonary Medicine, vol. 15, no. 3, pp. 188-200, 2009.

[93] K. Dheda, R. N. van Zyl-Smit, R. Meldau et al., "Quantitative lung $\mathrm{T}$ cell responses aid the rapid diagnosis of pulmonary tuberculosis," Thorax, vol. 64, no. 10, pp. 847-853, 2009.

[94] R. S. Garfein, R. Lozada, L. Liu et al., "High prevalence of latent tuberculosis infection among injection drug users in Tijuana, Mexico," International Journal of Tuberculosis and Lung Disease, vol. 13, no. 5, pp. 626-632, 2009.

[95] W. Jiang, L. Shao, Y. Zhang et al., "High-sensitive and rapid detection of Mycobacterium tuberculosis infection by IFN$\gamma$ release assay among HIV-infected individuals in BCGvaccinated area," BMC Immunology, vol. 10, article 31, 2009.

[96] S. S. J. Lee, Y. C. Liu, T. S. Huang et al., "Comparison of the interferon-gamma; release assay and the tuberculin skin test for contact investigation of tuberculosis in BCG-vaccinated health care workers," Scandinavian Journal of Infectious Diseases, vol. 40, no. 5, pp. 373-380, 2008.

[97] A. Machado Jr., K. Emodi, I. Takenami et al., "Analysis of discordance between the tuberculin skin test and the interferongamma release assay," International Journal of Tuberculosis and Lung Disease, vol. 13, no. 4, pp. 446-453, 2009.

[98] M. X. Rangaka, K. A. Wilkinson, R. Seldon et al., "Effect of HIV-1 infection on T-cell-based and skin test detection of tuberculosis infection," American Journal of Respiratory and Critical Care Medicine, vol. 175, no. 5, pp. 514-520, 2007.

[99] L. Richeldi, M. Losi, R. D’Amico et al., "Performance of tests for latent tuberculosis in different groups of immunocompromised patients," Chest, vol. 136, no. 1, pp. 198-204, 2009.

[100] I. Rivas, I. Latorre, A. Sanvisens et al., "Prospective evaluation of latent tuberculosis with interferon- $\gamma$ release assays in drug and alcohol abusers," Epidemiology and Infection, vol. 137, no. 9, pp. 1342-1347, 2009.

[101] M. Ruhwald, J. Petersen, K. Kofoed et al., "Improving T-cell assays for the diagnosis of latent TB infection: potential of a diagnostic test based on IP-10," PLoS ONE, vol. 3, no. 8, Article ID e2858, 2008.
[102] C. Stephan, T. Wolf, U. Goetsch et al., "Comparing QuantiFERON-tuberculosis gold, T-SPOT tuberculosis and tuberculin skin test in HIV-infected individuals from a low prevalence tuberculosis country," AIDS, vol. 22, no. 18, pp. 2471-2479, 2008.

[103] N. J. Talati, U. Seybold, B. Humphrey et al., "Poor concordance between interferon- $\gamma$ release assays and tuberculin skin tests in diagnosis of latent tuberculosis infection among HIV-infected individuals," BMC Infectious Diseases, vol. 9, article 15, 2009.

[104] B. Chang, H. Y. Park, K. Jeon, J. K. Ahn, H. S. Cha, E. M. Koh et al., "Interferon-gamma release assay in the diagnosis of latent tuberculosis infection in arthritis patients treated with tumor necrosis factor antagonists in Korea," Clinical Rheumatology, vol. 30, no. 12, pp. 1535-1541, 2011.

[105] E. Tavast, T. Tuuminen, S. H. Pakkanen, M. Eriksson, A. Kantele, A. Jarvinen et al., "Immunosuppression adversely affects TST but Not IGRAs in patients with psoriasis or inflammatory musculoskeletal diseases," International Journal of Rheumatology, vol. 2012, Article ID 381929, 8 pages, 2012.

[106] R. Smith, A. Cattamanchi, K. R. Steingart, C. Denkinger, K. Dheda, K. L. Winthrop et al., "Interferon-gamma release assays for diagnosis of latent tuberculosis infection: evidence in immune-mediated inflammatory disorders," Current Opinion in Rheumatology, vol. 23, no. 4, pp. 377-384, 2011.

[107] D. I. Ling, M. Pai, V. Davids, L. Brunet, L. Lenders, R. Meldau et al., "Are interferon-gamma release assays useful for diagnosing active tuberculosis in a high-burden setting?" European Respiratory Journal, vol. 38, no. 3, pp. 649-656, 2011.

[108] C. B. E. Chee, S. H. Gan, K. W. KhinMar et al., "Comparison of sensitivities of two commercial gamma interferon release assays for pulmonary tuberculosis," Journal of Clinical Microbiology, vol. 46, no. 6, pp. 1935-1940, 2008.

[109] L. Leidl, H. Mayanja-Kizza, G. Sotgiu et al., "Relationship of immunodiagnostic assays for tuberculosis and numbers of circulating CD4 ${ }^{+}$T-cells in HIV infection," European Respiratory Journal, vol. 35, no. 3, pp. 619-626, 2010.

[110] R. Markova, Y. Todorova, R. Drenska, I. Elenkov, M. Yankova, and D. Stefanova, "Usefulness of interferon-gamma release assays in the diagnosis of tuberculosis infection in HIV-infected patients in Bulgaria," Biotechnology and Biotechnological Equipment, vol. 23, no. 1, pp. 1103-1108, 2009.

[111] F. Bartalesi, S. Vicidomini, D. Goletti et al., "QuantiFERON-TB Gold and the TST are both useful for latent tuberculosis infection screening in autoimmune diseases," European Respiratory Journal, vol. 33, no. 3, pp. 586-593, 2009.

[112] S. M. Behar, D. S. Shin, A. Maier, J. Coblyn, S. Helfgott, and M. E. Weinblatt, "Use of the T-SPOT.TB assay to detect latent tuberculosis infection among rheumatic disease patients on immunosuppressive therapy," Journal of Rheumatology, vol. 36, no. 3, pp. 546-551, 2009.

[113] M. Bocchino, A. Matarese, B. Bellofiore et al., "Performance of two commercial blood IFN- $\gamma$ release assays for the detection of Mycobacterium tuberculosis infection in patient candidates for anti-TNF- $\alpha$ treatment," European Journal of Clinical Microbiology and Infectious Diseases, vol. 27, no. 10, pp. 907-913, 2008.

[114] N. Cobanoglu, U. Ozcelik, U. Kalyoncu et al., "Interferongamma assays for the diagnosis of tuberculosis infection before using tumour necrosis factor-alpha blockers," International Journal of Tuberculosis and Lung Disease, vol. 11, no. 11, pp. 1177-1182, 2007. 
[115] F. Gogus, Z. Günendi, R. Karakus, Z. Erdogan, K. Hizel, and F. Atalay, "Comparison of tuberculin skin test and QuantiFERON-TB gold in tube test in patients with chronic inflammatory diseases living in a tuberculosis endemic population," Clinical and Experimental Medicine, vol. 10, no. 3, pp. 173-177, 2010.

[116] S. Kleinert, O. Kurzai, J. Elias et al., "Comparison of two interferon- $\gamma$ release assays and tuberculin skin test for detecting latent tuberculosis in patients with immune-mediated inflammatory diseases," Annals of the Rheumatic Diseases, vol. 69, no. 4, pp. 782-784, 2010.

[117] A. J. Kwakernaak, P. M. Houtman, J. F. L. Weel, J. P. L. Spoorenberg, and T. L. T. A. Jansen, "A comparison of an interferon-gamma release assay and tuberculin skin test in refractory inflammatory disease patients screened for latent tuberculosis prior to the initiation of a first tumor necrosis factor $\alpha$ inhibitor," Clinical Rheumatology, vol. 30, no. 4, pp. 505-510, 2011.

[118] E. Laffitte, J. P. Janssens, P. Roux-Lombard et al., “Tuberculosis screening in patients with psoriasis before antitumour necrosis factor therapy: comparison of an interferon- $\gamma$ release assay vs. tuberculin skin test," British Journal of Dermatology, vol. 161, no. 4, pp. 797-800, 2009.

[119] C. D. L. Marques, A. L. B. P. Duarte, V. M. B. De Lorena et al., "Evaluation of an interferon gamma assay in the diagnosis of latent tuberculosis infection in patients with rheumatoid arthritis," Rheumatology International, vol. 30, no. 1, pp. 57-62, 2009.

[120] J. Martin, C. Walsh, A. Gibbs et al., "Comparison of interferon $\gamma$ release assays and conventional screening tests before tumour necrosis factor $\alpha$ blockade in patients with inflammatory arthritis," Annals of the Rheumatic Diseases, vol. 69, no. 1, pp. 181-185, 2010.

[121] G. Matulis, P. Jüni, P. M. Villiger, and S. D. Gadola, "Detection of latent tuberculosis in immunosuppressed patients with autoimmune diseases: performance of a Mycobacterium tuberculosis antigen-specific interferon $\gamma$ assay," Annals of the Rheumatic Diseases, vol. 67, no. 1, pp. 84-90, 2008.

[122] D. P. De Leon, E. Acevedo-Vasquez, S. Alvizuri et al., "Comparison of an interferon- $\gamma$ assay with tuberculin skin testing for detection of tuberculosis (TB) infection in patients with rheumatoid arthritis in a TB-endemic population," Journal of Rheumatology, vol. 35, no. 5, pp. 776-781, 2008.

[123] A. M. Schoepfer, B. Flogerzi, S. Fallegger et al., "Comparison of interferon-gamma release assay versus tuberculin skin test for tuberculosis screening in inflammatory bowel disease," American Journal of Gastroenterology, vol. 103, no. 11, pp. 2799-2806, 2008.

[124] D. Vassilopoulos, N. Stamoulis, E. Hadziyannis, and A. J. Archimandritis, "Usefulness of enzyme-linked immunosorbent assay (Elispot) compared to tuberculin skin testing for latent tuberculosis screening in rheumatic patients scheduled for anti-tumor necrosis factor treatment. Addendum," Journal of Rheumatology, vol. 35, no. 7, article 1464, 2008.

[125] G. H. Mazurek, J. Jereb, A. Vernon, P. LoBue, S. Goldberg, and K. Castros, "Updated guidelines for using interferon gamma release assays to detect Mycobacterium tuberculosis infection-United States, 2010," Morbidity and Mortality Weekly Report, vol. 59, no. RR05, pp. 1-25, 2010.

[126] H. Nakaoka, L. Lawson, S. B. Squire et al., "Risk for tuberculosis among children," Emerging Infectious Diseases, vol. 12, no. 9, pp. 1383-1388, 2006.
[127] K. Okada, T. E. Mao, T. Mori et al., "Performance of an interferon-gamma release assay for diagnosing latent tuberculosis infection in children," Epidemiology and Infection, vol. 136, no. 9, pp. 1179-1187, 2008.

[128] R. Petrucci, N. A. Amer, R. Q. Gurgel et al., "Interferon gamma, interferon-gamma-induced-protein 10, and tuberculin responses of children at high risk of tuberculosis infection," Pediatric Infectious Disease Journal, vol. 27, no. 12, pp. 1073-1077, 2008.

[129] T. A. Thomas, D. Mondal, Z. Noor et al., "Malnutrition and helminth infection affect performance of an interferon $\gamma$-release assay," Pediatrics, vol. 126, no. 6, pp. e1522-e1529, 2010.

[130] J. C. Choi, J. W. Shin, J. Y. Kim, I. W. Park, B. W. Choi, and M. K. Lee, "The effect of previous tuberculin skin test on the follow-up examination of whole-blood interferon- $\gamma$ assay in the screening for latent tuberculosis infection," Chest, vol. 133, no. 6, pp. 1415-1420, 2008.

[131] K. Ewer, K. A. Millington, J. J. Deeks, L. Alvarez, G. Bryant, and A. Lalvani, "Dynamic antigen-specific T-cell responses after point-source exposure to Mycobacterium tuberculosis," American Journal of Respiratory and Critical Care Medicine, vol. 174, no. 7, pp. 831-839, 2006.

[132] P. C. Hill, R. H. Brookes, A. Fox et al., "Longitudinal assessment of an ELISPOT test for Mycobacterium tuberculosis infection," PLoS Medicine, vol. 4, no. 6, pp. 1061-1070, 2007.

[133] M. Pai, R. Joshi, S. Dogra et al., "Serial testing of health care workers for tuberculosis using interferon- $\gamma$ assay," American Journal of Respiratory and Critical Care Medicine, vol. 174, no. 3, pp. 349-355, 2006.

[134] R. N. van Zyl-Smit, A. Zwerling, K. Dheda, and M. Pai, "Withinsubject variability of interferon-g assay results for tuberculosis and boosting effect of tuberculin skin testing: a systematic review," PLoS ONE, vol. 4, no. 12, Article ID e8517, 2009.

[135] T. Yoshiyama, N. Harada, K. Higuchi, Y. Nakajima, and H. Ogata, "Estimation of incidence of tuberculosis infection in health-care workers using repeated interferon- $\gamma$ assays," Epidemiology and Infection, vol. 137, no. 12, pp. 1691-1698, 2009.

[136] S. Machingaidze, S. Verver, H. Mulenga, D. A. Abrahams, M. Hatherill, W. Hanekom et al., "Predictive value of recent quantiferon conversion for tuberculosis disease in adolescents," American Journal of Respiratory and Critical Care Medicine, vol. 186, no. 10, pp. 1051-1056, 2012.

[137] S. Perry, L. Sanchez, S. Yang, Z. Agarwal, P. Hurst, and J. Parsonnet, "Reproducibility of QuantiFERON-TB gold in-tube assay," Clinical and Vaccine Immunology, vol. 15, no. 3, pp. 425-432, 2008.

[138] A. Schablon, M. Harling, R. Diel, F. C. Ringshausen, J. Torres Costa, and A. Nienhaus, "Serial testing with an interferongamma release assay in German healthcare workers," GMS Krankenhaushygiene Interdisziplinär, vol. 5, no. 2, 2010.

[139] D. Tripodi, B. Brunet-Courtois, V. Nael et al., "Evaluation of the tuberculin skin test and the interferon-release assay for TB screening in French healthcare workers," Journal of Occupational Medicine and Toxicology, vol. 4, no. 1, article 30, 2009.

[140] S. E. Van Brummelen, A. M. Bauwens, N. J. Schlösser, and S. M. Arend, "Kinetics of a tuberculosis-specific gamma interferon release assay in military personnel with a positive tuberculin skin test," Clinical and Vaccine Immunology, vol. 17, no. 6, pp. 937-943, 2010. 
[141] K. Higuchi, N. Harada, and T. Mori, "Interferon- $\gamma$ responses after isoniazid chemotherapy for latent tuberculosis," Respirology, vol. 13, no. 3, pp. 468-472, 2008.

[142] J. E. Kaplan, C. Benson, K. H. Holmes, J. T. Brooks, A. Pau, and H. Masur, "Guidelines for prevention and treatment of opportunistic infections in HIV-infected adults and adolescents: recommendations from CDC, the National Institutes of Health, and the HIV Medicine Association of the Infectious Diseases Society of America," Morbidity and Mortality Weekly Report. Recommendations and Reports, vol. 58, no. 4, Article ID CE1, p. 1, 2009. 


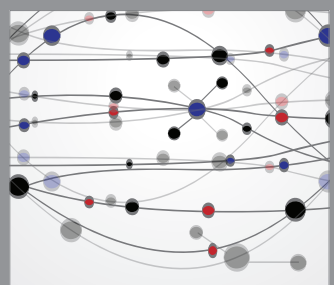

The Scientific World Journal
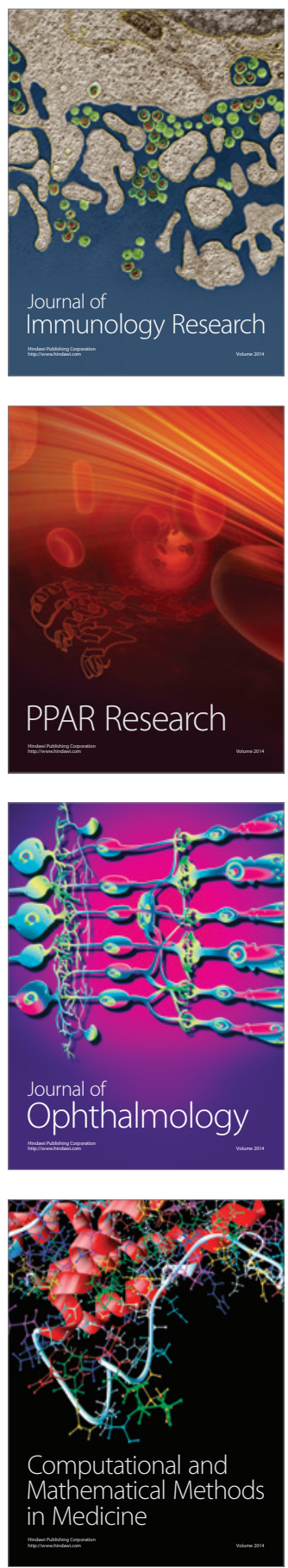

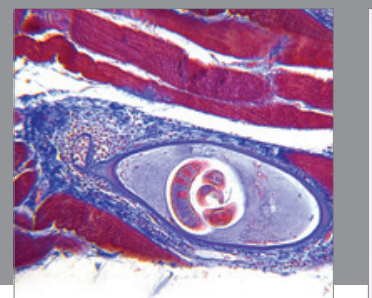

Gastroenterology

Research and Practice
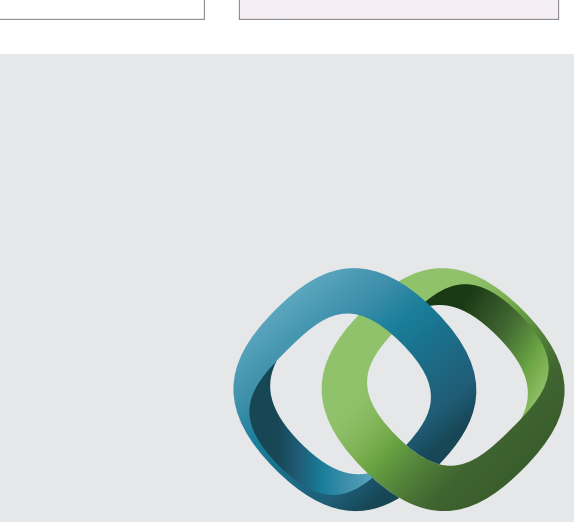

\section{Hindawi}

Submit your manuscripts at

http://www.hindawi.com
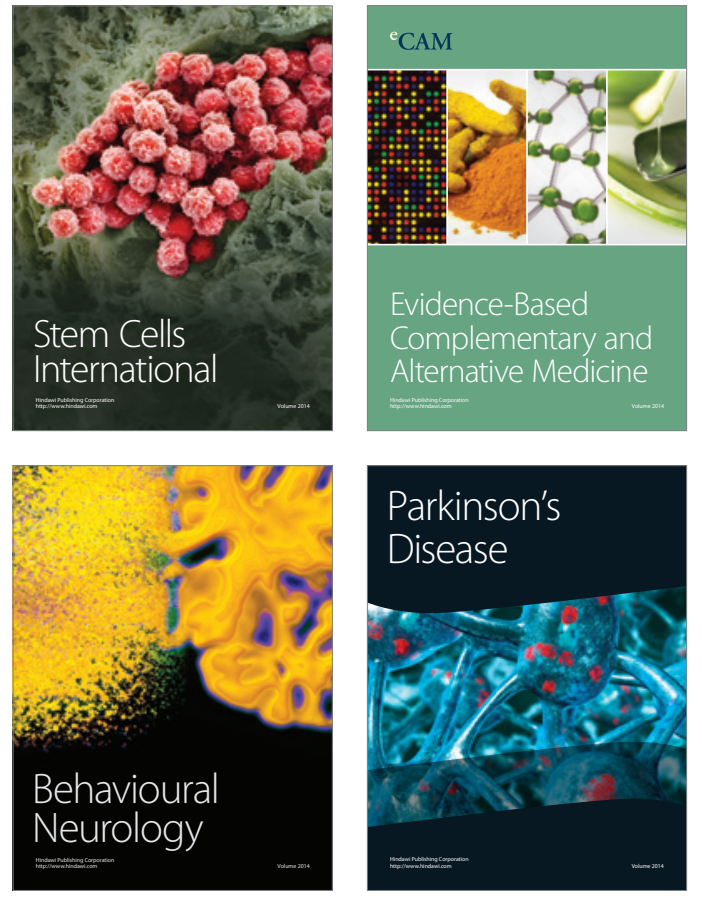
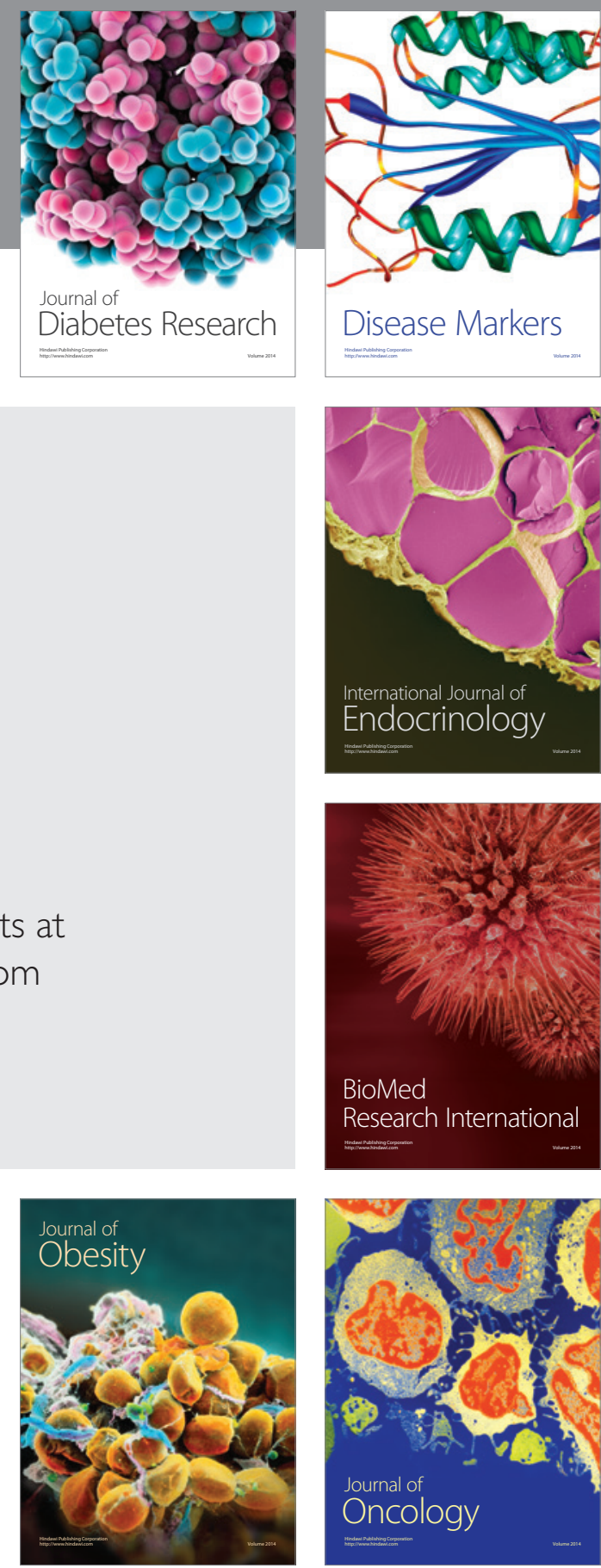

Disease Markers
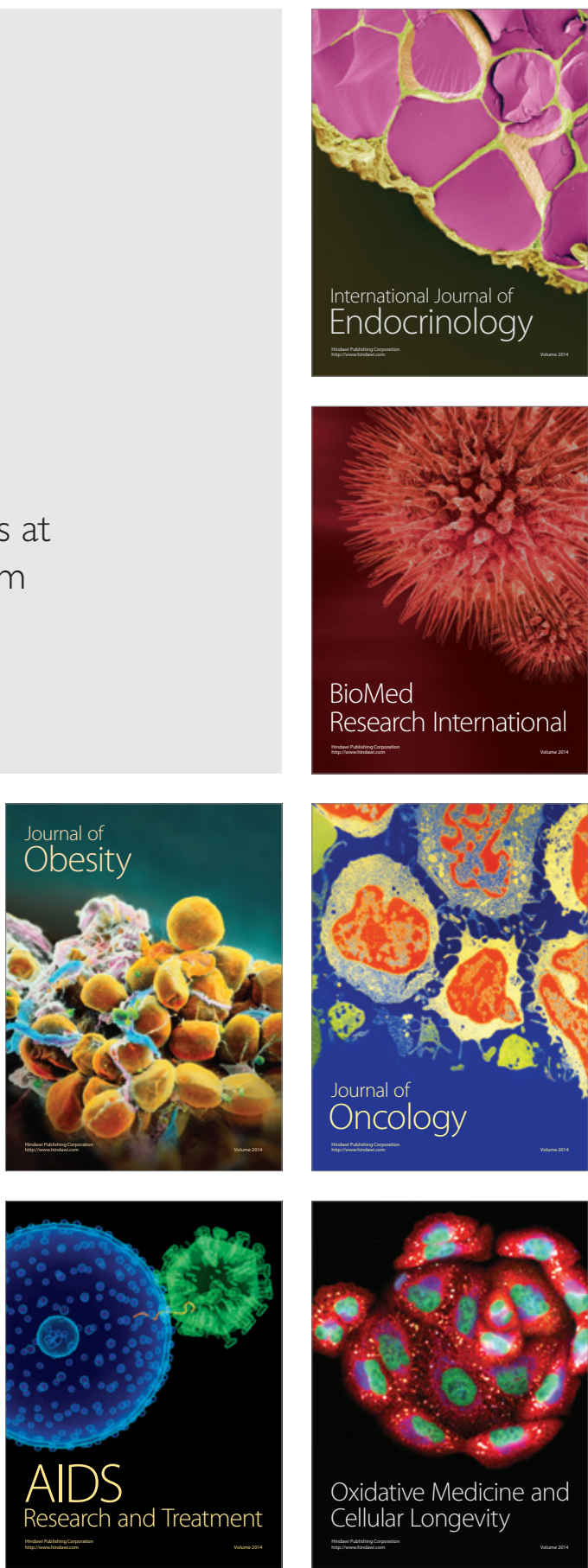